\title{
REVIEW
}

\section{REVIEW OF THE SPINAL TUBERCULOSIS SURGICAL TREATMENT AND OUTCOMES}

\author{
Sayed Abdulla Jami ${ }^{1}$, Shi Jiandang ${ }^{1}$, Zhanwen Zhou ${ }^{1}$, Liu Chang Hao ${ }^{1}$ \\ ${ }^{1}$ Department of Spinal Surgery, General Hospital of Ningxia Medical University, Ningxia, People's Republic \\ of China
}

Corresponding author: Shi Jiandang

Email: shi_jiandang@outlook.com

\begin{abstract}
Spinal tuberculosis (TB), caused by mycobacterium tuberculosis, is one of the oldest diseases in the world. Spinal TB represents 50\% of bone and joint TB. If spinal TB is not diagnosed and treated in time, it could cause spinal cord infection, nerve compression and paralysis. Spinal TB often causes abscesses formation, spinal dysfunction, spinal instability, and kyphosis onset disorder. Most spinal TB can be cured by non-surgical treatment. Surgery is the ultimate method of treatment of spinal tuberculosis and it is used for eliminating lesions, relieving spinal cord and nerve compression, correcting kyphosis and building spine stability. At present, spinal TB surgical approaches are mainly posterior approach, combined anterior-posterior approach and minimally invasive techniques. The present review summarizes the advantages, disadvantages and indications for each surgical method.
\end{abstract}

KEYWORDS: review, current, advance, surgical approaches, spinal tuberculosis

\section{INTRODUCTION}

Spinal tuberculosis (TB) can be localized in different sites of the vertebra. The pathological sites can be divided into central types and marginal types. The central type tends to occur in children, and marginal type tends to occur in adults. The development of spinal TB from the onset is significantly different from case to case. The most commonly affected spinal segments are thoracic vertebrae $(48.03 \%)$ and lumbar vertebrae $(42.36 \%)$ followed by thoraco-lumbar vertebrae $(29.58 \%)$, cervical vertebrae $(5.39 \%)$ [1]. Sacral vertebrae TB (4.22\%) is relatively rare [1]. In many parts of the developing countries, TB remains endemic and increases due to migration. A total of 1.3 million people dies annually from TB. Spinal TB represents about 1 to $2 \%$ of all cases of $\mathrm{TB}$, and it's the most common musculoskeletal manifestation of TB. Its common clinical manifestations include back pain, constitutional symptoms, spinal tenderness and deformities and paraplegia. This review study focuses on the various aspects of spinal TB, currently available surgical techniques, diagnosis development and the outcomes.

\section{MATERIALS AND METHOD}

We conducted a formal review of any published literature from the last 30 years related to spinal $\mathrm{TB}$ in internet-based journals and PubMed. This study was approved by the General Hospital of Ningxia Medical University committee for clinical research. 


\section{RESULTS}

\section{Diagnosis of Spinal Tuberculosis}

Imaging Examination

At present, imaging examination of spinal TB mainly includes X-ray, CT, MRI, and PET-CT. In recent years, thanks to the widespread of medical technology in hospitals at all levels, the accuracy of spinal tuberculosis diagnosis has steadily improved. Imaging techniques reveal if the vertebral body and intervertebral space are damaged. The disadvantage is the lack of early specific manifestation in spinal TB patients. There are 5 types of spinal tuberculosis CT findings [2]: bone fragment type, osteolytic type, focal destruction sclerosis type, subperiosteal type and mixed type. CT scans have a significant role in establishing the treatment plan.

MRI is highly sensitive to changes in water and proteins in tissues. MRI shows intervertebral disc involvement, lesion range, abscess formation, intraspinal lesions and degree of nerve compression.

PET-CT is a non-invasive examination. After intravenous injection of $18 \mathrm{~F}-\mathrm{FDG}$ (fluorodeoxyglucose), CT examination was used as an auxiliary system. 18F-FDG PET combined with CT is nowadays getting more and more relevant for the diagnosis of several infectious and inflammatory diseases. 18F-FDG-PET scan with CT component images the whole body and provides a metabolic map of the infection, enabling clinicians to assess the disease burden and see tissues or cells changes on affected area. $18 \mathrm{~F}-\mathrm{FDG}$ uptake is not specific for tuberculosis infection, but multiple studies are currently showing that PET-CT scan could differentiate spinal TB from other inflammatory disease of the spine. It can also monitor anti-tuberculosis drug effects and can identify atypical spinal tuberculosis [3].

\section{Examination}

Laboratory and Histopathological

Patients with spinal tuberculosis are often accompanied by ESR (erythrocyte sedimentation rate) and elevated C-reactive protein, normal routine blood test, occasionally decreased neutrophil percentage, increased lymphocyte percentage. However, the above tests lack specificity and are difficult to relate to the non- specific spine infection of brucellosis spondylitis. In recent years, the T-spot test (ELISA spot test) has become an important method of diagnosis for spinal tuberculosis. Tspot test is fast, has high sensitivity and can be used as an essential auxiliary diagnostic method before the bacteriological examination is clear. The gold standard are histopathology and bacteriological examination. For atypical spinal $\mathrm{TB}$, an abscess or a diseased vertebra puncture can be performed under the guidance of ultrasound, X-ray, or CT. Qualitative PCR cannot determine whether Mycobacterium tuberculosis is viable or not. New RNA tests for Mycobacterium tuberculosis are being developed in order to diagnose TB infections.

\section{Preoperative Drug Treatment}

Standardized preoperative anti-TB treatment is necessary for the treatment of spinal TB. Anti TB drugs inhibits the growth of mycobacterium tuberculosis in the lesion, reduces the bacilli number and also controls the presence of mycobacterium tuberculosis in the body. Preoperative anti-TB drugs are Isoniazid, rifampicin, ethambutol, and pyrazinamide in quadruple classic combination [4-5], but for antiTB preoperative medicines, there are still some controversy over the duration of treatment. Most authors believe that the preoperative treatment period should last 2 to 4 weeks [4, 6]. Yin et al. [7] reported more than 31 cases with segmental thoracic tuberculosis treated preoperatively with anti-TB drugs for 3 - 17 days; all patients average 6.6 days, no spread of mycobacterium tuberculosis occurred, the kyphosis was corrected and excellent bone graft fusion was obtained. Mycobacterium tuberculosis drug resistance was not evident in patients with preoperative anti-TB drugs. Surgery can be performed if ESR, CRP, and the patient's overall condition improve $[4,8]$.

Indications for surgical treatment of spinal tuberculosis [4, 7, 9] are: (1) vertebral junction visible structural damage, secondary spinal instability; (2) compression of the spinal cord and cauda equina nerves or paraplegia; (3) presence of large dead bones, cavities, and lesions abscess; (4) severe or progressive protrusion deformity; (5) ineffective non-surgical treatment; (6) Drug resistance. Besides, to save neural function, Mak et al. [10] proposed the following indications for 
emergency spinal tuberculosis surgery: (1) progressive nerve function deterioration; (2) progressive spinal deformity; (3) non -surgical treatment failure; (4) severe pain; (5) Difficult diagnosis by microscopic examinations.

\section{DISCUSSION}

Treatment of spinal TB includes nonsurgical treatment and surgical treatment, including open surgery and minimally invasive surgery. Minimally invasive surgery is used for cervical tuberculosis, but there are only a few literature data about the treatment for this spinal segment. The reason may be that the brachial plexus nerves near the cervical spine are relatively dense and a micro-invasive surgery could easily cause vascular and nerve damage. For spinal tuberculosis, the first choice of surgical treatment or non-hand treatment (minimally invasive) is controversial. The main purposes of spinal tuberculosis surgery are to remove dead bones and abscesses, correct spinal shape deformities, relieve nerve compression and relieve pain. With the development of anti-TB drugs as well as the improvement of drug treatment programs, the effect of non-surgical treatment is constantly improving. Anti-TB drug treatment is always the basis of spinal TB treatment. The surgical treatment is also an important treatment for the rapid recovery of the spine. So, in this review, study we focused on the development and current surgical direction of spinal TB treatments.

\section{Surgical Approach}

\section{Anterior Approach}

The majority of the TB lesion in the spine are located in the vertebral body. The anterior approach allows direct entry into the tuberculosis lesion and can be done under direct vision; afterward, decompression and bone graft reconstruction are performed $[11,12]$.

Liu et al. [13] treated 60 patients with spinal tuberculosis (30 cases of thoracolumbar tuberculosis and 30 cases of lumbar tuberculosis); the patients were studied in groups according to three surgical methods used (10 cases of thoracic and lumbar tuberculosis in each group). The authors showed that vertebrae reconstruction rate in anterior approach was 1.9 times higher than posterior approach and anterior-posterior combined approach.

Zeng et al. [14] present 59 patients with cervical and thoracic spinal TB who underwent surgery by anterior approach (20 cases), posterior approach (21 cases), and anterior-posterior combined approach (18 cases). The author found that the anterior approach is significantly inferior regarding the kyphotic angle correction rate than posterior approach and combined anteriorposterior approach. Hassan et al. [15] and Liu et al. [13] have the same results. Ling et al. [16] also pointed out that the ability of anterior surgery to correct kyphosis was limited, and the preoperative kyphosis Cobb Angle of spinal tuberculosis treated by anterior surgery should be $<30^{\circ}$.

After consulting the relevant literature and clinical data for the past ten years, we observed that the indications for anterior approach are as follows [12, 13, 14]: (1) lesion mainly located in the front column; (2) Severe collapse of vertebral body requiring reconstruction of vertebral height; (3) large prevertebral abscess; (4) necrotic tissue infection and compression of the front part of the spinal cord; (5) no severe kyphosis. At the same time, the exposure range and trauma during the anterior surgery are large, so it is necessary to improve the protection awareness of the thoracic and abdominal organs. In order to avoid postoperative complications such as atelectasis, pleural effusion, spread of mycobacterium tuberculosis in the chest and abdomen, damage to the great vessels and ureters.

\section{Posterior Approach}

Although the anterior approach is effective in removing lesions and reconstructing vertebral height has advantages. However, with the widespread use of pedicle screws and with the development of technology, the posterior approach has gradually become spinal tuberculosis mainstream surgical treatment.

Liu et al. [17] performed a singlesegment thoracic and lumbar spine posterior debridement bone grafting and pedicle screw internal fixation in 37 cases ( 7 in the thoracic spine, 25 in the thoracolumbar spine and 5 in the lumbar spine); bone graft fusion was achieved in all patients on average 5.6 months after surgery, the Cobb angle improved from $28.30^{\circ} \pm 11.95^{\circ}$ 
before surgery to $5.50^{\circ} \pm 11.84^{\circ}$ after surgery and at the last follow-up was $7.20^{\circ} \pm 11.56^{\circ}$; regarding neural function (Frankel Grade classification) [18], 3 cases of grade A had no change, and the remaining patients were all improved by grade A - C after surgery. After the operation 2 patients developed intercostal neuralgia and 1 patient developed superficial infection around the incision.

Hassan et al. [15] reported 42 patients with single-segment thoracic and lumbar spinal tuberculosis who underwent surgical intervention (anterior approach in 20 cases and posterior approach in 22 cases); It was found that the average operation time, blood loss and blood transfusion volume in the anterior approach were significantly lower than in the posterior approach; the capacity. of posterior approach to correct kyphosis was significantly higher than anterior approach and the loss of correction angle was also lower. The posterior approach also has advantages in reducing back pain. The indications for posterior approach surgery are [5, 8]: (1) lesion limited to a single segment or to 2 adjacent segments; (2) relatively localized abscess or small tuberculosis lesion that could be completely removed; (3) history of several anterior approaches that disorganized the local anatomy. Also, we believe that if the main damage is in the posterior spinal column with severe spinal canal damage, stenosis and severe spinal cord compression or severe patients with pulmonary infections, pleural adhesions and abdominal organ diseases, posterior approach is preferred.

\section{Approaches}

Combined Anterior - Posterior

Anterior and posterior approaches can be combined during the same procedure. Through anterior resection, the lesion can be removed and the vertebral height reconstructed, while posterior approach can be used to correct kyphosis and to fix the bone graft with pedicle screws.

Wang et al. [19] made a retrospective analysis of 184 thoracic vertebral TB cases in adult patients comparing three surgical approaches used; the Cobb angle in the anteriorposterior combined approach group (65 patients) was corrected from $29.3^{\circ} \pm 9.1^{\circ}$ before surgery to $7.0^{\circ} \pm 1.1^{\circ}$ after surgery, and the last follow- up it measured $8.6^{\circ} \pm 1.7^{\circ}$. In terms of correcting kyphosis, there was no significant difference between combined approach and posterior approach. The anterior approach is inferior to the combined approach regarding the correction of kyphosis. All three methods equally improve nerve function and fusion time of bone graft. Combined anterior and posterior approaches require two operations, so this method inevitably leads to longer operative times and more intraoperative outages of blood volume and postoperative complications. after the analysis of relevant literature and clinical practice, we conclude that the indications for anteriorposterior combined approach are: (1) damaged anterior structure of the vertebral column with severe kyphosis; (2) multiple segmental spinal tuberculosis; (3) extensive paravertebral abscesses, fluid abscesses and necrotic bone; (4) Difficulties in performing anterior fixation or loss of anterior fixation. Besides, due to the combined approach before and after surgery trauma, the process phase for complex, the author recommends for the elderly patients, and the basic situation is that poor patients should be used with caution. Highly skilled surgeons should apply the technique.

\section{Reconstruction Methods \\ Bone Graft Fusion}

Surgical treatment for spinal tuberculosis involves removing the lesion and bone defects, reconstructing the ridge stability of the column using bone graft fusion and partially correcting the kyphosis of the spine. The types of grafts that can be utilized include autogenous bone grafts, allogeneic bone grafts and synthetic grafts (metal implants). Autogenous bone is the best bone graft material, mainly from the autogenous iliac crest, rib and fibular bone [11, 12].

Compared with allogeneic and artificial bone, autogenous bone has better biocompatibility and low immune rejection [20]. He et al. [11] suggests that the autogenous ilium is preferred because of its blood vessels and rapid ossification rate, but the patient's donor site may develop complications in up to $10 \%$ of the cases. Chronic pain at the donor site occurs in nearly $40 \%$ of the cases [21].

Allogeneic bone graft is mainly obtained from the frozen femur, humerus or iliac crest and can be used in order to avoid donor site 
complications. Yin et al. [22] conducted a retrospective analysis of 25 patients with spinal TB who received circular allograft bone treatment; all patients who underwent surgery received bone graft fusion within 3 months after surgery. All wounds healed well and no graft rejection occurred.

Some surgeons currently choose metal implants (titanium mesh) to play the role of the scaffold. Also, metal implants can be mixed with bone grafting materials such as autogenous bone; studies have found that titanium mesh can provide strong structural support, has a good bone graft fusion rate and is efficient in restoring vertebral height [7]. Gao et al. [27] found that for single-segment in spinal tuberculosis, titanium mesh can achieve the same clinical effect as autogenous ilium bone graft. In the treatment of multi-segment spinal tuberculosis titanium mesh may be more efficient [23]. Metal implants used to stabilize the defect in tuberculosis lesion of the spine has been controversial because bacteria can grow on the surface of the implant, producing polysaccharides/protein membranes in order to evade the body's immune response and resisting the bactericidal action of anti-TB drugs [24]. These pathogenic processes can cause fusion failure and relapse. However, some studies have shown that the adhesion of bacteria is very low, and the use of titanium mesh will not cause the spread of infection. This may also be associated with standard drug therapy, thorough debridement and focal disease severity related to factors such as the patient's underlying condition. On the other hand, the use synthetic grafts eliminate complications after autologous bone transplantation and immune rejection of allograft bone, but it should be associated with thorough debridement and standardized drug therapy. Titanium mesh should be used for patients with relatively good health. Factors such as patient willingness and hospital conditions also need to be taken into account.

\section{Internal Fixations}

Patients with spinal tuberculosis need to undergo internal fixation to increase the joints stability after bone grafting. Internal fixation promotes bone graft fusion and prevents tuberculosis recurrence. At present, the main internal fixation methods are long-segment fixation (at least 2 motor units fixed across the affected vertebrae), short segment fixation (1 above and 1 below the affected vertebra fixed motor units) and single-segment fixation (for single-segment spinal tuberculosis - it is only fixed within the lesion segment). A single segment fixation retains more spinal motor function $[25,26]$. The indications and of singlesegment fixation [31] are :(1) a lesion involving only a single motor segment, (2) possibility of inserting internal fixation devices between adjacent vertebral bodies, (3) bone grafts placed through the anterior approach; Contraindications of single-segment fixation [31]: (1) kyphosis Angle $>30^{\circ}$, (2) bone osteoporosis, (3) osteotomy required for spinal TB. D'Souza et al. [32] made a retrospective analysis of 21 cases of thoracic or thoracolumbar spine TB treated with posterior decompression and fixation; the kyphosis angle was corrected from $21.61^{\circ} \pm 3.72^{\circ}$ preoperative to $5.79^{\circ} \pm 3.48^{\circ}$ after surgery, with a value at the last follow up of $8.74^{\circ} \pm 3.65^{\circ}$; the nerve function of the patients was restored. The current pedicle screw technique for the posterior fixation of spinal tuberculosis is widely used. It can also correct bone graft stability, kyphosis deformity, it reduces patient's healing time and bedtime and it improves the high spinal tuberculosis cure rate.

\section{Minimally Invasive Surgery}

Spinal TB can be treated with minimally invasive surgery. In recent years, minimally invasive techniques for the spine developed rapidly and can be performed through thoracoscopy or laparoscopy; percutaneous puncture was performed with a minimally invasive approach. In spinal TB surgical procedures are for debridement, drug infusion, bone graft fusion and internal fixation. Minimally invasive surgery provides are safe and have reduced complications. Garg et al. [27] reviewed and analyzed 22 cases of minimally invasive surgical treatment of spinal tuberculosis; the function was improved in different degrees and complications occurred in 2 cases after surgery; thoracic and lumbar kyphosis angles were corrected from $13.3^{\circ} \pm 4.3^{\circ}$ before surgery to $32.8^{\circ} \pm 2.8^{\circ}$. Lumbar kyphosis angles were corrected from $10.8^{\circ} \pm 2.8^{\circ}$ before surgery to $24.3^{\circ} \pm 10^{\circ}$ after surgery; at the last follow-up mean value was $12.8^{\circ}$ and $27.0^{\circ}$ respectively. Lü et al. [28] reported 50 cases of thoracic spinal tuberculosis patients who 
underwent minimally invasive surgery. The kyphosis angel was corrected from $30.2^{\circ} \pm 0.2^{\circ}$ to $18.5^{\circ} \pm 8.5^{\circ}$; the correction rate was $38.7 \%$, and the mean loss at the last follow-up was $1.3 \%$.

On the other side, compared to the correction rate of convex deformity after open surgery (using the 3 approaches), the one after minimally invasive surgery is relatively inadequate. Also, minimally invasive surgery cannot be applied if the patient has pleural adhesion, respiratory dysfunction or has already underwent open surgery. Moreover, minimally invasive techniques have a steep learning curve. The low correction rate of convex deformity leads to its unpopularity. Therefore, we believe that for patients who meet the conditions, minimally invasive surgery can be properly carried out by a surgeon who masters the technique.

\section{CONCLUSION}

Surgical treatment of patients with spinal tuberculosis needs to be individualized and associated preoperatively with anti-TB drugs. Each of the three conventional techniques has its advantages and disadvantages. There is no such "universal technique" in clinical practice. In choosing a surgical method, surgeons should consider its advantages, disadvantages, indications, flexibly, costs, patient willingness, hospital resources and other factors. However, with the widespread application and improvement of minimally invasive technology in various clinical disciplines, it will definitely make a breakthrough in the treatment of spinal tuberculosis.

\section{REFERENCES}

[1] T. Shi et al., "Retrospective Study of 967 Patients with Spinal Tuberculosis," Orthopedics, vol. 39, no. 5, pp. e838-e843.

[2] R. Jain, S. Sawhney, and M. Berry, "Computed tomography of vertebral tuberculosis: patterns of bone destruction," vol. 47, no. 3, p. 196, 1993.

[3] S. Dureja, I. B. Sen, and S. Acharya, "Potential role of F18 FDG PET-CT as an imaging biomarker for the noninvasive evaluation in uncomplicated skeletal tuberculosis: a prospective clinical observational study," (in eng), Eur Spine J, vol. 23, no. 11, pp. 2449-54, Nov 2014.
[4] X. Wang, X. Pang, P. Wu, C. Luo, and X. Shen, "One-stage anterior debridement, bone grafting and posterior instrumentation vs. single posterior debridement, bone grafting, and instrumentation for the treatment of thoracic and lumbar spinal tuberculosis," European Spine Journal, vol. 23, no. 4, pp. 830-837.

[5] Z. Xu et al., "Posterior only versus combined posterior and anterior approaches for lower lumbar tuberculous spondylitis with neurological deficits in the aged," Spinal Cord, vol. 53, no. 6, pp. 482-487.

[6] X. Shen, H. Liu, G. Wang, and X. Liu, "Singlestage posterior-only approach treating single-segment thoracic tubercular spondylitis," International Journal of Clinical \& Experimental Pathology, vol. 8, no. 9, pp. 11051-11059, 2015.

[7] X. H. Yin et al., "The role of costotransverse radical debridement, fusion and postural drainage in the surgical treatment of multisegmental thoracic spinal tuberculosis: a minimum 5-year follow-up," European Spine Journal, vol. 25, no. 4, pp. 10471055.

[8] J Liu, L Wan, X Long, et al. Efficacy and safety of posterior versus combined posterior and anterior approach for the treatment of spinal tuberculosis : a meta-analysi $[\mathrm{J}]$. World Neurosurg, 2015, 83 (6) : 1157-1165.

[9] A. Jain, I. Dhammi, B. Prashad, S. Sinha, and P. Mishra, "Simultaneous anterior decompression and posterior instrumentation of the tuberculous spine using an anterolateral extrapleural approach," The Journal of bone and joint surgery. British volume, vol. 90, no. 11 , pp. 1477-1481, 2008.

[10] K. C. Mak and K. M. C. Cheung, "Erratum to: Surgical treatment of acute TB spondylitis: indications and outcomes," European Spine Journal, vol. 22, no. 1, pp. 211-211.

[11] M. He, H. Xu, J. Zhao, and Z. Wang, "Anterior debridement, decompression, bone grafting, and instrumentation for lower cervical spine tuberculosis," Spine Journal, vol. 14, no. 4, pp. 619627.

[12] Li et al., "One-stage surgical management for thoracic tuberculosis by anterior debridement, decompression and autogenous rib grafts, and instrumentation," vol. 11, no. 8, pp. 726-733, 2011. [13] P. Liu, M. Sun, S. Li, Z. Wang, and G. Ding, "A retrospective controlled study of three different operative approaches for the treatment of thoracic and lumbar spinal tuberculosis: Three years of followup," Clinical Neurology \& Neurosurgery, vol. 128, pp. 25-34.

[14] H. Zeng et al., "Comparison of three surgical approaches for cervicothoracic spinal tuberculosis: a retrospective case-control study," vol. 10, no. 1, pp. 1-10, 2015. 
[15] K. Hassan and E. Elmorshidy, "Anterior versus posterior approach in surgical treatment of tuberculous spondylodiscitis of thoracic and lumbar spine," European Spine Journal, vol. 25, no. 4, pp. 1056-1063.

[16] T. Ling, L. Liu, X. Yang, Z. Qiang, X. Hu, and Y. An, "Revision surgery for spinal tuberculosis with secondary deformity after treatment with debridement, instrumentation, and fusion," European Spine Journal, vol. 24, no. 3, pp. 577-585.

[17] Z. Liu, L. Jiaming, P. Aifeng, L. Xinhua, Y. Dong, and H. Shanhu, "One-Stage Posterior Debridement and Transpedicular Screw Fixation for Treating Monosegmental Thoracic and Lumbar Spinal Tuberculosis in Adults," The Scientific World Journal, vol. 2014, pp. 1-6.

[18] H. L. Frankel et al., "The value of postural reduction in the initial management of closed injuries of the spine with paraplegia and tetraplegia," vol. 7, no. 3, pp. 179-192.

[19] L. Wang, H. Zhang, M. Tang, Q. Gao, Z. Zhou, and $\mathrm{X}$. Yin, "Comparison of Three Surgical Approaches for Thoracic Spinal Tuberculosis in Adult," vol. 42, no. 11, pp. 808-817, 2017.

[20] H. Q. Zhang et al., "One-stage posterior debridement, transforaminal lumbar interbody fusion and instrumentation in treatment of lumbar spinal tuberculosis: a retrospective case series," Archives of Orthopaedic \& Trauma Surgery, vol. 133, no. 3, pp. 333-341, 2013.

[21] H. Zhang, K. Zeng, X. Yin, J. Huang, M. Tang, and C. Guo, "Debridement, internal fixation, and reconstruction using titanium mesh for the surgical treatment of thoracic and lumbar spinal tuberculosis via a posterior-only approach: a 4-year follow-up of 28 patients," Journal of Orthopaedic Surgery \& Research, vol. 10, no. 1, p. 150.

[22] X. Yin, P. Liu, Y.-y. Liu, W.-L. Fan, B.-y. Liu, and J.-H. Zhao, "Utilization of ring-shaped bone allograft for surgical treatment of adolescent posttubercular kyphosis," Medicine, vol. 96, no. 24, p. e7132.

[23] Y. Gao, Y. Ou, Q. Deng, B. He, X. Du, and J. Li, "Comparison between titanium mesh and autogenous iliac bone graft to restore vertebral height through posterior approach for the treatment of thoracic and lumbar spinal tuberculosis," (in eng), PLoS One, vol. 12, no. 4, p. e0175567, 2017.

[24] H. Ky, Y. Chung, and S. Ryoo, "Adherence and biofilm formation of Staphylococcus epidermidis and Mycobacterium tuberculosis on various spinal implants," Spine, vol. 30, no. 1, pp. 38-43, 2005.

[25] Z. Wang, H. Yuan, G. Geng, J. Shi, and W. Jin, "Posterior mono-segmental fixation, combined with anterior debridement and strut graft, for treatment of the mono-segmental lumbar spine tuberculosis," vol. 36, no. 2, pp. 325-329.
[26] Z. Wang, Q. Wu, and G. Geng, "Anterior debridement and bone grafting with posterior singlesegment internal fixation for the treatment of monosegmental spinal tuberculosis," Injury, vol. 44, no. 2, pp. 253-257, 2013.

[27] N. Garg and R. Vohra, "Minimally invasive surgical approaches in the management of tuberculosis of the thoracic and lumbar spine," Clinical Orthopaedics and Related Research ${ }^{\circledR}$, vol. 472, no. 6, pp. 1855-1867, 2014.

[28] G. Lü, B. Wang, J. Li, W. Liu, and I. Cheng, "Anterior debridement and reconstruction via thoracoscopy-assisted mini-open approach for the treatment of thoracic spinal tuberculosis: minimum 5year follow-up," vol. 21, no. 3, pp. 463-469. 